\title{
Joint Source-Channel Coding for the MIMO Broadcast Channel
}

\author{
Daniel Persson, Johannes Kron, Mikael Skoglund and Erik G. Larsson
}

\section{Linköping University Post Print}

N.B.: When citing this work, cite the original article.

C2012 IEEE. Personal use of this material is permitted. However, permission to reprint/republish this material for advertising or promotional purposes or for creating new collective works for resale or redistribution to servers or lists, or to reuse any copyrighted component of this work in other works must be obtained from the IEEE.

Daniel Persson, Johannes Kron, Mikael Skoglund and Erik G. Larsson, Joint Source-Channel Coding for the MIMO Broadcast Channel, 2012, accepted IEEE Transactions on Signal Processing.

Postprint available at: Linköping University Electronic Press

http://urn.kb.se/resolve?urn=urn:nbn:se:liu:diva-73221 


\title{
Joint Source-Channel Coding for the MIMO Broadcast Channel
}

\author{
Daniel Persson ${ }^{\dagger *}$, Johannes Kron ${ }^{\ddagger}$, Mikael Skoglund ${ }^{\ddagger}$, and Erik G. Larsson ${ }^{\dagger}$ \\ ${ }^{\dagger}$ Mailing address: Daniel Persson, Erik G. Larsson, Department of Electrical Engineering, \\ Linköping University, SE-581 83 Linköping, Sweden \\ Email: $\quad$ danielp@isy.liu.se, egl@isy.liu.se \\ Phone: $\quad$ +46 $\quad$ + $\quad$ 13281351, +46 13281312 \\ Fax: $\quad$ +46 13139282
}

${ }^{\ddagger}$ Mailing address: Johannes Kron, Mikael Skoglund, School of Electrical Engineering, Royal Institute of Technology, Osquldas väg 10, SE-100 44 Stockholm, Sweden

Email: $\quad$ johk@ee.kth.se, skoglund@ee.kth.se

Phone: $\quad$ +4687908415, +4687908430

Fax: $\quad+4687907260$

* Corresponding author

SOFTWARE: $\quad$ Ubuntu Linux, TeX, BibTeX, Kile

COPYRIGHT: $\quad$ Copyright (c) 2011 IEEE. Personal use of this material is permitted. However, permission to use this material for any other purposes must be obtained from the IEEE by sending a request to pubs-permissions@ieee.org.

\footnotetext{
Abstract: We investigate the problem of broadcasting analog sources to several users using short codes, employing several antennas at both the transmitter and the receiver, and channel-optimized quantization. Our main objective is to minimize the sum mean square error distortion. A joint multi-user encoder, as well as a structured encoder with separate encoders for the different users, are proposed. The first encoder outperforms the latter, which in turn offers large improvements compared to state-of-the-art, over a wide range of channel signal-to-noise ratios. Our proposed methods handle bandwidth expansion, i.e., usage of more channel than source dimensions, automatically. We also derive a lower bound on the distortion.
} 


\title{
Joint Source-Channel Coding for the MIMO Broadcast Channel
}

\author{
Daniel Persson, Johannes Kron, Mikael Skoglund, and Erik G. Larsson
}

\begin{abstract}
We investigate the problem of broadcasting analog sources to several users using short codes, employing several antennas at both the transmitter and the receiver, and channel-optimized quantization. Our main objective is to minimize the sum mean square error distortion. A joint multi-user encoder, as well as a structured encoder with separate encoders for the different users, are proposed. The first encoder outperforms the latter, which in turn offers large improvements compared to state-of-the-art, over a wide range of channel signal-to-noise ratios. Our proposed methods handle bandwidth expansion, i.e., usage of more channel than source dimensions, automatically. We also derive a lower bound on the distortion.
\end{abstract}

\section{Index Terms}

Short codes, multiple-input multiple-output (MIMO), multi-user, broadcast, downlink, joint sourcechannel coding (JSCC), low latency.

\section{INTRODUCTION}

We study the multiple-input multiple-output (MIMO) broadcast problem with short codes, with the end-to-end sum mean square error (MSE) distortion as performance measure. This investigation can be motivated by low-latency applications, where long codes are not suitable. Our study is both of fundamental interest, and relevant for applications.

D. Persson and E. G. Larsson are with the Department of Electrical Engineering, Linköping University, SE-581 83 Linköping, Sweden (e-mail: danielp@isy.liu.se; egl@isy.liu.se).

J. Kron and M. Skoglund are with the School of Electrical Engineering, Royal Institute of Technology, Osquldas väg 10, SE-100 44 Stockholm, Sweden (e-mail: johk@ee.kth.se; skoglund@ee.kth.se).

This work has been supported in part by the Swedish Research Council (VR) and the Swedish Foundation for Strategic Research (SSF). E.G. Larsson is a Royal Swedish Academy of Sciences (KVA) Research Fellow supported by a grant from the Knut and Alice Wallenberg Foundation. 


\section{A. Background}

MIMO technology improves both capacity and robustness in traditional communications [1]. Recently, Weingarten et al. [2] found the capacity region for the MIMO broadcast channel, and it was established that dirty-paper coding (DPC) is optimal in the sense that techniques based on DPC can achieve any rate-tuple in this region. However, DPC relies on infinitely long codewords, and is therefore not suitable for a low-delay scenario.

In a scenario with short codes, the source-channel separation theorem in general does not apply, and the optimal solution consists of joint optimization of the source and the channel code. Traditional digital communication systems consist of several separate units: quantizer, channel encoder, precoder, and modulator. Joint source-channel coding (JSCC) strategies, where these units are co-designed, should be considered for the case of short codes.

Many treatments of JSCC exist, e.g., characterization of the distortion regions for the problems of sending a bivariate Gaussian source over bandwidth-matched Gaussian broadcast channels [3] and multiple-access channels [4], information-theoretic analysis of separation of source and channel coding over several multiuser channels [5], transmission over non-ergodic channels based on instantaneous capacity-achieving codes [6], distortion-exponents-based analysis for high channel signal-to-noise ratio (CSNR) [7], linear source-channel mappings for the broadcast channel [8], and other parametric mappings for Gaussian multiple-access relay channels [9].

Channel-optimized quantization is a way to perform JSCC, that does not depend on the assumptions of instantaneous capacity-achieving codes, high CSNR approximations, and linear and other mapping parametrizations. This is a tandem approach where channel code usage and modulation format are first decided, and where the quantizer, and the mapping of quantizer indexes to channel codewords, are optimized in a second step depending on the channel transition probabilities determined after the first step. The algorithm in [10] determines channel-optimized vector quantizer reconstruction vectors and encoding regions, for single-user communications. This algorithm can be seen as an extension to noisy channels of the generalized Lloyd algorithm (GLA) [11]. A benefit of the algorithm in [10] is that it chooses the tradeoff between quantization distortion and channel-induced distortion automatically, and it can thus be seen as an adaptive quantization and channel coding scheme.

Numerous extensions of the scheme in [10] have been found. Some recent developments are, e.g., the study of mappings of a scalar source to several channel dimensions, i.e., bandwidth expansion [12], channel-optimized source mappings for the relay channel [13], and channel-optimized quantization for 
sources with memory [14], [15]. Some of these schemes were intended for traditional digital communications [12], [14], [15], while [13] focuses on analog communication. This is however only a matter of presentation of the works, and all schemes based on [10] are valid for both digital and analog domainbased communications. More modulation points result in a lower source reconstruction distortion, but also a higher computational complexity.

\section{B. Our scope and contribution}

In contrast to the channel-optimized quantization approaches stemming from [10] and described in Sec. I-A, we study channel-optimized quantization for interfering multi-user channels in this paper. More precisely, we investigate the MIMO broadcast scenario, where different streams are simultaneously sent to different users, and where all streams interfere at each user. The employed codes are short, and we utilize several antennas at both the transmitter and the receiver sides. Our main objective is to minimize the sum end-to-end distortion.

Our JSCC schemes employ the vector perturbation precoder (VPP) scheme [16] as a building block. The basic idea of VPP is to reduce power consumption through expanding the original signaling constellation. VPP has been documented to reduce the error probability significantly compared to linear methods and scalar Tomlinson-Harashima precoding [17], see [16]. However, our proposed JSCC schemes can deal with MIMO interference by proper modeling of the transition probabilities between codewords, for any precoder, modulation, and detector scheme. Our proposed methods handle bandwidth expansion, i.e., usage of more channel than source dimensions, automatically.

Our main contributions are:

- We propose a joint multi-user encoder which outperforms the methods of comparison. This encoder has exponential complexity in the number of users.

- A transmitter with separate encoders for the different users, and linear complexity in the number of users, is developed. Despite being based on the separate user approximation, this encoder lowers distortion significantly compared to state-of-the-art. In our experiments we further note that the proposed JSCC approach contributes more to lowering the distortion than the VPP approach.

- We derive a lower bound on the distortion.

\section{MIMO BROADCAST TRANSMISSION WITH SHORT CODES}

In this section, we present two new optimized low-latency coding strategies for communication over a MIMO broadcast channel, as well as a derivation of a lower bound on the distortion. Real-valued 


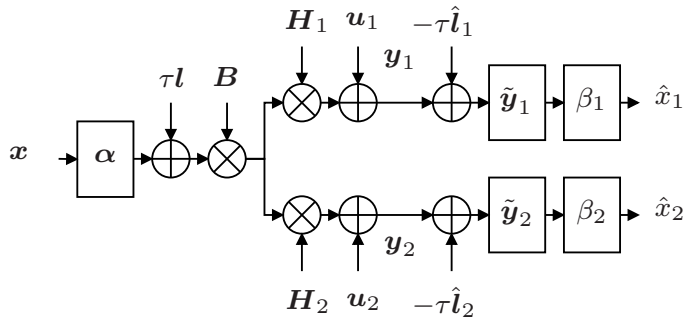

(a)

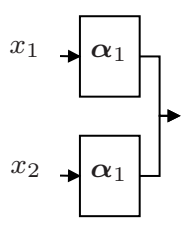

(b)

Fig. 1. In (a), the proposed JSCC system is pictured. In (b), the proposed simplified encoder in Sec. II-B is shown.

signaling per antenna is considered in this paper for simplicity. However, complex-valued signaling can be represented in this format, by appropriately treating a complex number as a vector of two real numbers. Our goal is to minimize the sum end-to-end MSE distortion. We assume perfect channel state information (CSI) at the transmitter and at the receivers. For each channel realization, a new coder has to be designed. In order to keep the notation simple, we consider two users, see Fig. 1(a). It is conceptually straightforward to generalize the developments to any number of terminals, though no fundamental aspects are added by such an extension.

The source variables $x_{1} \in \mathbb{R}$ and $x_{2} \in \mathbb{R}$ are independent and identically distributed (i.i.d.) zeromean Gaussian with unit variance (a non-zero mean is handled by simply removing the mean at the transmitter and adding it back at the receiver), and will be delivered to users 1 and 2 respectively. We define $\boldsymbol{x}=\left[x_{1}, x_{2}\right]^{\mathrm{T}}$.

User $i$ 's channel consists of a mixing matrix $\boldsymbol{H}_{i} \in \mathbb{R}^{N_{\mathrm{R}} \times N_{\mathrm{T}}}$, and additive noise $\boldsymbol{u}_{i} \in \mathbb{R}^{N_{\mathrm{R}}}$ whose components are i.i.d. Gaussian with zero mean and unit variance, for $i=1,2$, where $N_{\mathrm{T}}$ is the total number of transmit antennas, and $N_{\mathrm{R}}$ is the number of receive antennas per user. We also write $\boldsymbol{H}=$ $\left[\boldsymbol{H}_{1}^{T} \boldsymbol{H}_{2}^{T}\right]^{T}$ and $\boldsymbol{u}=\left[\boldsymbol{u}_{1}^{T} \boldsymbol{u}_{2}^{T}\right]^{T}$.

We define our analog communication system as follows. We use VPP [16] with the precoding matrix $\boldsymbol{B}=\left[\boldsymbol{B}_{1} \boldsymbol{B}_{2}\right]$, where $\boldsymbol{B}_{i} \in \mathbb{R}^{N_{\mathrm{T}} \times N_{\mathrm{R}}}$, for $i=1,2$. Following the original development in [16], we constrain $\boldsymbol{B}$ to being of regularized inverse-type $\boldsymbol{B}=\boldsymbol{H}^{T}\left(\boldsymbol{H} \boldsymbol{H}^{T}+\eta \frac{N_{T}}{P} \boldsymbol{I}\right)^{-1}$, where $P$ is the mean total transmit power per realization of $\boldsymbol{x}$, and $\eta \in[0, \infty)$. The encoder mapping is $\boldsymbol{\alpha}(\boldsymbol{x}): \mathbb{R}^{2} \rightarrow \mathbb{R}^{2 N_{\mathrm{R}}} \cap$ $\Omega^{2 N_{\mathrm{R}}}$, where $\Omega^{2 N_{\mathrm{R}}}$ is a $2 N_{\mathrm{R}}$-dimensional cube with side less than $\tau$, centered at origo. The perturbation vectors are $\tau \boldsymbol{l}(\boldsymbol{\alpha})$, with $\boldsymbol{l}(\boldsymbol{\alpha}) \in \mathcal{L}$, and $\mathcal{L}=\left\{\boldsymbol{l}: l_{k} \in\{-L,-L+1, \ldots, L\}\right.$ for $\left.k=1, \ldots, 2 N_{\mathrm{R}}\right\}$, obtained by calculating $\boldsymbol{l}^{*}(\boldsymbol{\alpha})=\underset{\boldsymbol{l}^{\prime} \in \mathcal{L}}{\arg \min }\left\|\boldsymbol{B}\left(\boldsymbol{\alpha}(\boldsymbol{x})+\tau \boldsymbol{l}^{\prime}\right)\right\|^{2}$. Finally, we write the received signals as $\boldsymbol{y}=\boldsymbol{H} \boldsymbol{B}(\boldsymbol{\alpha}(\boldsymbol{x})+\tau \boldsymbol{l}(\boldsymbol{\alpha}))+\boldsymbol{u}$, where $\boldsymbol{y}=\left[\boldsymbol{y}_{1}^{T} \boldsymbol{y}_{2}^{T}\right]^{T}$, and $\boldsymbol{y}_{i} \in \mathbb{R}^{N_{\mathrm{R}}}$, for $i=1,2$.

Estimates $\hat{\boldsymbol{l}}_{1}=\left[\hat{l}_{1}, \ldots, \hat{l}_{N_{\mathrm{R}}}\right]^{T}$ of the elements $l_{1}, \ldots, l_{N_{\mathrm{R}}}$ are retrieved and subtracted at user 1 's decoder, 
and estimates $\hat{l}_{2}=\left[\hat{l}_{N_{\mathrm{R}}+1}, \ldots, \hat{l}_{2 N_{\mathrm{R}}}\right]^{T}$ of the elements $l_{N_{\mathrm{R}}+1}, \ldots, l_{2 N_{\mathrm{R}}}$ are retrieved and subtracted at user 2's decoder. The standard estimates [16] are

$$
\hat{l}_{k}=\left\lfloor\frac{y+\frac{(\boldsymbol{H} \boldsymbol{B})_{k, k} \tau}{2}}{(\boldsymbol{H} \boldsymbol{B})_{k, k} \tau}\right\rfloor, k=1, \ldots, 2 N_{\mathrm{R}},
$$

where $\lfloor\cdot\rfloor$ is the flooring operation. The decoder mappings are $\beta_{i}\left(\boldsymbol{y}_{i}\right): \mathbb{R}^{N_{\mathrm{R}}} \cap \Omega^{N_{\mathrm{R}}} \rightarrow \mathbb{R}$, for $i=1,2$. Transmitting $x_{1}$ over $\boldsymbol{H}_{1} \boldsymbol{B}_{1}$ is motivated by $\boldsymbol{B}$ being of regularized inverse-type, so that $\boldsymbol{H}_{1} \boldsymbol{B}_{1}$ contains more energy than $\boldsymbol{H}_{1} \boldsymbol{B}_{2}$ (this reasoning is similar for $x_{2}$ ).

It is difficult to optimize $\boldsymbol{B}$ and $\tau$ in order to minimize the sum end-to-end distortion, and simple closed form expressions for $\boldsymbol{B}$ and $\tau$ may not exist. We defer a more detailed discussion on how we set $\boldsymbol{B}, \tau$, and $L$ to Sec. III-A.

We further assume a mean power constraint

$$
\mathrm{E}_{\boldsymbol{x}}\left[\|\boldsymbol{B}(\boldsymbol{\alpha}(\boldsymbol{x})+\tau \boldsymbol{l}(\boldsymbol{\alpha}))\|^{2}\right] \leq P,
$$

which is fulfilled by assuming $\boldsymbol{\alpha}$ to be uniformly distributed over $\Omega^{2 N_{\mathrm{R}}}$, and adjusting the size of $\Omega^{2 N_{\mathrm{R}}}$, prior to optimizing the mappings $\boldsymbol{\alpha}(\boldsymbol{x}), \beta_{1}\left(\boldsymbol{y}_{1}\right)$, and $\beta_{2}\left(\boldsymbol{y}_{2}\right)$.

The rest of this section will be dedicated to the design of optimized source-channel mappings $\boldsymbol{\alpha}(\boldsymbol{x})$, $\beta_{1}\left(\boldsymbol{y}_{1}\right)$, and $\beta_{2}\left(\boldsymbol{y}_{2}\right)$, given this channel-coding scheme. We define a cost function

$$
\begin{aligned}
& J\left(\boldsymbol{\alpha}(\boldsymbol{x}), \beta_{1}\left(\boldsymbol{y}_{1}\right), \beta_{2}\left(\boldsymbol{y}_{2}\right)\right) \\
& =\mathrm{E}_{\boldsymbol{x}, \boldsymbol{u}_{1}, \boldsymbol{u}_{2}}\left[\frac{1}{2} \sum_{i=1}^{2}\left(x_{i}-\beta_{i}\left(\boldsymbol{y}_{i}\right)\right)^{2}\right] \\
& =\mathrm{E}_{\boldsymbol{x}, \boldsymbol{y}_{1}, \boldsymbol{y}_{2}}\left[\frac{1}{2} \sum_{i=1}^{2}\left(x_{i}-\beta_{i}\left(\boldsymbol{y}_{i}\right)\right)^{2}\right] .
\end{aligned}
$$

We thus want to solve

$$
\begin{aligned}
&\left\{\boldsymbol{\alpha}^{*}(\boldsymbol{x}), \beta_{1}^{*}\left(\boldsymbol{y}_{1}\right), \beta_{2}^{*}\left(\boldsymbol{y}_{2}\right)\right\} \\
& \quad=\underset{\left\{\boldsymbol{\alpha}(\boldsymbol{x}), \beta_{1}\left(\boldsymbol{y}_{1}\right), \beta_{2}\left(\boldsymbol{y}_{2}\right)\right\}}{\arg \min } J\left(\boldsymbol{\alpha}(\boldsymbol{x}), \beta_{1}\left(\boldsymbol{y}_{1}\right), \beta_{2}\left(\boldsymbol{y}_{2}\right)\right) .
\end{aligned}
$$

Throughout our treatment, we will continue to use $(\cdot)^{*}$ to denote optimal mappings. The optimization problem in (5) is very complicated. More precisely, the optimal mappings could be general non-linear mappings with no closed-form solution, and it is difficult to achieve both the encoder and the decoders simultaneously. We therefore employ a sub-optimal strategy, namely, we introduce a discretization of the 
channel space, and solve iteratively for the optimal encoders given the decoders, and for the optimal decoders given the encoders, in Sec. II-A and II-B.

We first discretize $\Omega^{2 N_{\mathrm{R}}}$ with $M$ points along each dimension $\mathcal{S}=\left\{\left(m-\frac{M-1}{2}\right) \Delta, m=0, \ldots, M-1\right\}$, and restrict $\boldsymbol{\alpha}(\boldsymbol{x}): \mathbb{R}^{2} \rightarrow \mathcal{S}^{2 N_{\mathrm{R}}}$. Moreover, $\boldsymbol{y}_{i}$ is least squares-decoded to yield $\tilde{\boldsymbol{y}}_{i}$

$$
\tilde{\boldsymbol{y}}_{i}=\underset{\boldsymbol{s} \in \mathcal{S}^{N_{\mathrm{R}}}}{\arg \min }\left\|\boldsymbol{y}_{i}-\boldsymbol{H}_{i} \boldsymbol{B}_{i}\left(\boldsymbol{s}+\tau \hat{\boldsymbol{l}}_{i}\right)\right\|^{2}, i=1,2 .
$$

The decoder (6) is a maximum likelihood (ML) decoder in the limit where the non-Gaussian MIMO interference is weak, and where $\tau$ is big enough compared to the size of $\mathcal{S}$ and the noise power, so that $\boldsymbol{l}$ is seldom mis-detected. The standard tandem approach from [16] that we have applied here, where $\hat{\boldsymbol{l}}_{i}$ is first estimated by (1), and where $\tilde{\boldsymbol{y}}_{i}$ is subsequently obtained by (6), could be replaced by a joint estimation of $\hat{\boldsymbol{l}}_{i}$ and $\tilde{\boldsymbol{y}}_{i}$, for $i=1,2$.

The decoder mappings $\boldsymbol{\beta}_{i}$ now operate on $\tilde{\boldsymbol{y}}_{i}, \boldsymbol{\beta}_{i}\left(\tilde{\boldsymbol{y}}_{i}\right): \mathcal{S}^{N_{\mathrm{R}}} \rightarrow \mathbb{R}$ for $i=1,2$. As long as $\Delta^{2}$ is small compared to the noise variance, and $M$ is big enough so that $\mathcal{S}^{2 N_{\mathrm{R}}}$ spans over the whole volume of $\Omega^{2 N_{\mathrm{R}}}$, the discretization is a good approximation [13]. We describe how to choose $\Delta$ in Sec. III-A in order to meet the mean power constraint (2).

We now rewrite (5) as

$$
\begin{aligned}
& \left\{\boldsymbol{\alpha}^{*}(\boldsymbol{x}), \beta_{1}^{*}\left(\tilde{\boldsymbol{y}}_{1}\right), \beta_{2}^{*}\left(\tilde{\boldsymbol{y}}_{2}\right)\right\} \\
& \quad=\underset{\left\{\boldsymbol{\alpha}(\boldsymbol{x}), \beta_{1}\left(\tilde{\boldsymbol{y}}_{1}\right), \beta_{2}\left(\tilde{\boldsymbol{y}}_{2}\right)\right\}}{\arg \min } J\left(\boldsymbol{\alpha}(\boldsymbol{x}), \beta_{1}\left(\tilde{\boldsymbol{y}}_{1}\right), \beta_{2}\left(\tilde{\boldsymbol{y}}_{2}\right)\right) .
\end{aligned}
$$

We note that while the state-of-the-art VPP detector with (1) and (6) is sub-optimal, the optimization problem (7) takes transitions probabilities between codewords, which incorporate the MIMO interference, as well as artifacts from the channel decoding by means of (1) and (6), into consideration. We can view our discretization approach as a digital communication system.

As already stated in the discussion following (5), with the discretization in (7), it is still difficult to obtain both the encoder and the decoders simultaneously. Therefore, we further introduce two suboptimal strategies. In Sec. II-A, we present a joint consideration of the two users at the encoder, and no other approximations than the iterative Lloyd approach that consists of encoder optimization for a given decoder, and decoder optimization for a given encoder. In Sec. II-B, we further approximate by separating the joint multi-user encoder from Sec. II-A into individual encoders for the users in order to lower the computational complexity. 


\section{A. Joint multi-user encoder}

Channel transition probabilities between transmit and receive constellation points are first determined by means of Monte Carlo simulation, and the decoders are initialized by $\beta_{i}^{(0)}$ at iteration step 0 , for $i=1,2$. Thereafter, at iteration step $k, k=1, \ldots, K$, the optimal encoder mapping $\boldsymbol{\alpha}^{(k)}$ for given decoder mappings $\beta_{i}^{(k-1)}$, and optimal decoder mappings $\beta_{i}^{(k)}$ for given encoder mappings $\boldsymbol{\alpha}^{(k)}$, are decided by means of

$$
\begin{aligned}
& \boldsymbol{\alpha}^{(k)}(\boldsymbol{x})=\underset{\boldsymbol{s} \in \mathcal{S}^{2 N_{\mathrm{R}}}}{\arg \min } \sum_{i=1}^{2} \mathrm{E}_{\tilde{\boldsymbol{y}}_{i} \mid \boldsymbol{s}}\left[\left(x_{i}-\beta_{i}^{(k-1)}\left(\tilde{\boldsymbol{y}}_{i}\right)\right)^{2}\right], \\
& \beta_{i}^{(k)}\left(\tilde{\boldsymbol{y}}_{i}\right)=\mathrm{E}_{x_{i} \mid \tilde{\boldsymbol{y}}_{i}}\left[x_{i}\right], \quad i=1,2 .
\end{aligned}
$$

The update equations (8) and (9) yield lower distortion at each iteration step $k, k=1, \ldots, K$. The iterative approach may however end up at a local minimum, and does not necessarily solve (7). When solving (9) in practice, the expectation is substituted by the empirical mean. Encoding by means of (8) in the general multi-user case with $N_{\mathrm{U}}$ users requires on the order of $N_{\mathrm{U}} M^{\left(N_{\mathrm{U}}+1\right) N_{\mathrm{R}}}$ operations. Since this number does not scale well with $N_{\mathrm{U}}$, we will propose a low-complexity method in the next section.

\section{B. Separate encoders for the different users}

We separate the problem (7) by constraining the source mapping $\boldsymbol{\alpha}(\boldsymbol{x})$ to have two independent parts $\boldsymbol{\alpha}(\boldsymbol{x})=\left[\boldsymbol{\alpha}_{1}^{T}\left(x_{1}\right) \boldsymbol{\alpha}_{2}^{T}\left(x_{2}\right)\right]^{T}$ where $\boldsymbol{\alpha}_{i}\left(x_{i}\right): \mathbb{R} \rightarrow \mathcal{S}^{N_{\mathrm{R}}}$ for $i=1,2$, see Fig. 1(b). The receive mappings are defined as before. Ideally, we would now like to solve the problem

$$
\begin{aligned}
& \left\{\boldsymbol{\alpha}_{1}\left(x_{1}\right), \boldsymbol{\alpha}_{2}\left(x_{2}\right), \beta_{1}^{*}\left(\tilde{\boldsymbol{y}}_{1}\right), \beta_{2}^{*}\left(\tilde{\boldsymbol{y}}_{2}\right)\right\} \\
& =\underset{\left\{\boldsymbol{\alpha}_{1}\left(x_{1}\right), \boldsymbol{\alpha}_{2}\left(x_{2}\right), \beta_{1}\left(\tilde{\boldsymbol{y}}_{1}\right), \beta_{2}\left(\tilde{\boldsymbol{y}}_{2}\right)\right\}}{\arg \min } \\
& J\left(\left[\boldsymbol{\alpha}_{1}^{T}\left(x_{1}\right) \boldsymbol{\alpha}_{2}^{T}\left(x_{2}\right)\right]^{T}, \beta_{1}\left(\tilde{\boldsymbol{y}}_{1}\right), \beta_{2}\left(\tilde{\boldsymbol{y}}_{2}\right)\right),
\end{aligned}
$$

but again, as with (7), the global optimal solution is hard to achieve. Instead, we solve (10) iteratively for the optimal encoders given the decoders, and for the optimal decoders given the encoders, by use of the equations

$$
\begin{aligned}
& \boldsymbol{\alpha}_{i}^{(k)}(\boldsymbol{x})=\arg \min _{\boldsymbol{s} \in \mathcal{S}^{N_{\mathrm{R}}}} \mathrm{E}_{\tilde{\boldsymbol{y}}_{i} \mid \boldsymbol{s}}\left[\left(x_{i}-\beta_{i}^{(k-1)}\left(\tilde{\boldsymbol{y}}_{i}\right)\right)^{2}\right], \\
& \beta_{i}^{(k)}\left(\tilde{\boldsymbol{y}}_{i}\right)=\mathrm{E}_{x_{i} \mid \tilde{\boldsymbol{y}}_{i}}\left[x_{i}\right],
\end{aligned}
$$


for $\mathrm{i}=1,2$, where (11) and (12) reduces distortion at each iteration step $k$, for $k=1, \ldots, K$. Similarly to the algorithm in Sec. II-A, the iterative approach may however end up in a local minimum, and does not necessarily solve (10).

For encoding by means of (11) in the general multi-user case with $N_{\mathrm{U}}$ users, the computational complexity increases as $N_{\mathrm{U}} M^{2 N_{\mathrm{R}}}$, which is linear in $N_{\mathrm{U}}$ instead of exponential, cf. the encoder (8) in Sec. II-A.

It should also be noted that both proposed encoders have an extra complexity term $(2 L+1)^{N_{\mathrm{U}} N_{\mathrm{R}}}$ stemming from the VPP encoding, as well as a complexity contribution stemming from the calculation of transition probabilities, which is exponential in the number of users. Differently from the encoder, which is defined for an analog $x$, these terms are straightforward to pre-process in slow fading.

\section{A lower bound on the distortion}

In order to obtain a lower bound on the distortion, we employ the source-channel separation theorem, calculate the maximum sum-rate, and distribute this rate among the sources in an optimal manner. ${ }^{1}$ The concept of uplink-downlink duality [20], [21] is employed, and the convex optimization problem

$$
\begin{aligned}
& R^{*}=\max _{\boldsymbol{S}_{1}, \boldsymbol{S}_{2}} \frac{1}{4} \log _{2}\left(\left|\boldsymbol{I}+\boldsymbol{H}^{\mathrm{T}}\left[\begin{array}{cc}
\boldsymbol{S}_{1} & \mathbf{0} \\
\mathbf{0} & \boldsymbol{S}_{2}
\end{array}\right] \boldsymbol{H}\right|\right), \\
& \boldsymbol{S}_{1}, \boldsymbol{S}_{2} \text { symmetric and positive definite, } \\
& \operatorname{Tr}\left(\boldsymbol{S}_{1}\right)+\operatorname{Tr}\left(\boldsymbol{S}_{2}\right) \leq \mathrm{P},
\end{aligned}
$$

is solved in order to find the maximum rate $R^{*}$ per user, in bits per channel use. Observing that the optimal rate allocation to two i.i.d. sources is equal, and that the maximum rate that can be conveyed over the channel is $2 R^{*}$, we have the bound $J \geq 2^{-2 R^{*}}$. This bound is not achievable in general, since we are not able to distribute the rate in (13) equally to the two users. A related result, a characterization of the achievable distortion region for the problem of sending two correlated scalar Gaussian sources over a bandwidth-matched Gaussian broadcast channel, was recently presented in [3].

\footnotetext{
${ }^{1}$ The source-channel separation theorem does not hold when we have correlated sources. This is so because the interference becomes lower when the correlation between the sources increases [18, p. 71], [19, p. 448]. In our case, we have uncorrelated sources.
} 


\section{EXPERIMENTS}

\section{A. Experimental prerequisites}

The experiment parameters are chosen as follows, in order to supply relevant experimental settings, and not favor any scheme:

- Proposed systems: Assuming that $\alpha$ is equidistributed over the constellation points, the distance between the constellation points in one dimension, $\Delta$, is adjusted by bisection so that the mean energy of the VPP constellation is $P \pm 0.005 P$, cf. (2).

As already discussed in Sec. II, simple closed form expressions for $\boldsymbol{B}$ and $\tau$ that minimize the sum end-to-end distortion may not exist. The MMSE precoding matrix, i.e., $\eta=1$, was chosen for the experiments presented in the figures, cf. [22]. With ZF precoding, i.e., $\eta=0$, our proposed JSCC schemes in Sec. II-A and Sec. II-B performed the same since there is no multi-user interference, and they still increased performance compared to the methods of comparison, though the MMSE precoder results were better. For simplicity, we chose $\tau$ by assuming that $\boldsymbol{\alpha}$ is equidistributed over the constellation points, that only $\boldsymbol{\alpha}$ on the edges of the constellation contributes to misdetection of the perturbation vector, and that the interference is Gaussian and independent for each dimension, and we obtain $\tau=\max _{i}\left(\frac{2 \sigma_{i}}{(\boldsymbol{H} \boldsymbol{B})_{i, i}} Q^{-1}\left(\frac{M}{2}\left(1-\left(1-P_{\text {Err }}\right)^{\frac{1}{2 N_{\mathrm{R}}}}\right)\right)\right)+(M-1) \Delta$, where $\sigma_{i}^{2}=$ $\frac{P}{2 N_{\mathrm{R}}} \sum_{j=1, j \neq i}^{2 N_{\mathrm{R}}}(\boldsymbol{H} \boldsymbol{B})_{i, j}^{2}+1$. The error probability associated with a misdetected perturbation vector is set to be less than $P_{\text {Err }}=10^{-6}$. Preliminary investigations showed that increasing $L$ beyond 1 did not lower distortion significantly.

Channel transitions probabilities for use with the channel-optimized coders are calculated by means of Monte Carlo simulations, taking MIMO interference as well as artifacts from the channel decoding by means of (1) and (6), into account. Monte Carlo simulations are run with 10000 vectors per possible realization of $\boldsymbol{\alpha}$ for the case of $N_{\mathrm{T}}=2$ and $N_{\mathrm{R}}=1$; and with 300 vectors per possible realization of $\boldsymbol{\alpha}$ for the case of $N_{\mathrm{T}}=4$ and $N_{\mathrm{R}}=2$.

Initial $\beta_{1}$ and $\beta_{2}$ are chosen by generating decoder source codebooks with GLA, and mapping these codewords to constellation points ordered lexicographically according to their dimensions. For coder optimization with (8) and (9), as well as with (11) and (12), a training set that is different from the evaluation set and consisting of 15000 realizations, is used. The number of iterations $K=15$. In the experiments with a MIMO channel of dimension $N_{\mathrm{T}}=2$ and $N_{\mathrm{R}}=1$, we use $M=32$; and for the experiments with $N_{\mathrm{T}}=4$ and $N_{\mathrm{R}}=2$, we use $M=16$.

- Source and channel evaluation data: The elements of $\boldsymbol{H}$ are i.i.d. Gaussian with zero mean and 
unit variance. We consider slow fading channels. In particular, $\boldsymbol{H}$ is constant for 15000 consecutive realizations of $\boldsymbol{x}$. Results are calculated as means over channel realizations, which are evaluated in the Monte-Carlo sense using 90 realizations of $\boldsymbol{H}$. The channel signal-to-noise ratio (CSNR) is defined as the mean transmitted power $P$ divided by the mean noise power.

The code used for generating the results can be found at [23].

\section{B. Results}

The main purpose of Fig. 2(a) is to show a comparison between the proposed joint multi-user coder in Sec. II-A and the proposed low-complexity system with separate coders for all users in Sec. II-B for $L=0$ and $L=1$. We use a MIMO channel with $N_{\mathrm{T}}=2$, and $N_{\mathrm{R}}=1$. A linear ZF-based analog, i.e., quantization-free, coder with MMSE receivers and its theoretical performance prediction, and the lower bound from Sec. II-C, are added as well for comparison.

We see that the multi-user coder in Sec. II-A with $L=1$ outperforms the other coders, for a large range of CSNR values. We can also conclude that the improvement given by the multi-user coder in Sec. II-A compared to the system with separate coders in Sec. II-B is bigger than the improvement from using VPP with $L=1$. The relatively large gap to the lower bound is explained by the inability of the short codes to achieve capacity and rate-distortion optimality.

The purpose of Fig. 2(b) is to show how the system with separate coders for all users in Sec. II-B compares with state-of-the-art systems in a scenario with more antennas, $N_{\mathrm{T}}=4$, and $N_{\mathrm{R}}=2$. A comparison is given between the system in Sec. II-B for $L=0$ and $L=1$; a standard separate source-channel coding (SSCC) approach with GLA-based vector quantization, largest minimum Hamming distance-optimal linear block codes with different rates (the number of information and channel bits are given for each method in the figure), Gray mapping of bits to symbols, VPP with $L=1$, and 16 constellation points per real dimension; analog ZF-precoder-based repetition coding with MMSE receivers, and its theoretical performance prediction; as well as the lower bound from Sec. II-C. One should further note that the range of optimal separate coding schemes with varying block code length constitutes an adaptive coding scheme.

The proposed low-complexity system with separate coders for all users from Sec. II-B and $L=1$ outperforms the separate coding approaches and the repetition coder, for a wide range of CSNR values. By comparing the proposed JSCC system with $L=0$ and the SSCC systems, we note that JSCC contributes more than VPP to improving the solution. 


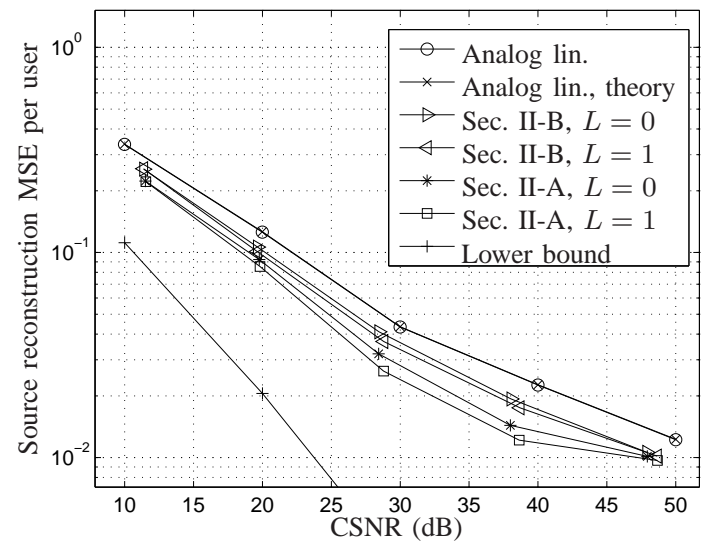

(a) $N_{\mathrm{T}}=2$, and $N_{\mathrm{R}}=1$.

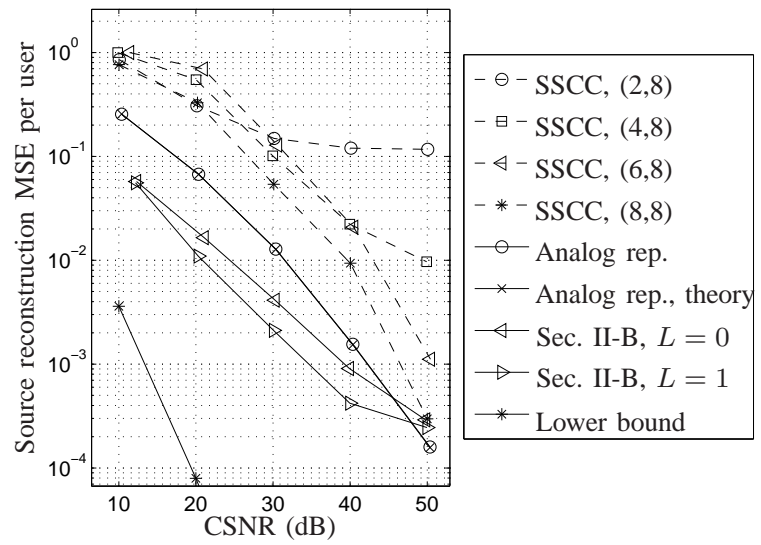

(b) $N_{\mathrm{T}}=4$, and $N_{\mathrm{R}}=2$.

Fig. 2. Comparisons between proposed schemes in Sec. II-A, Sec. II-B, and other methods, for different channel signal-to-noise ratios (CSNR). Details of the compared methods are given in the main text.

The repetition coder does not use a quantizer, and thus, it beats all other methods at extremely high CSNR. At extremely high CSNR, the separate source and channel coder system without a block-code, and the JSCC system with $L=0$, perform as well as the JSCC system with $L=1$. This should be so, since only the source encoder matters, and our proposed scheme becomes the traditional GLA algorithm, in this regime.

We observe that the measured CSNR may deviate from the target CSNR related to $P$. The reason for this is that some transmit constellation points are chosen more often than others. This practical issue could be taken care of by feeding a modified mean energy target to the $\Delta$-optimization.

\section{CONCLUSION}

We have studied source transmission over the MIMO broadcast channel, using short codes and with end-to-end sum-MSE as the performance measure. Two channel-optimized quantization-type systems, one with a joint multi-user encoder, and one with separate encoders for the different users, were proposed. The first encoder, which has exponential computational complexity in the number of users, outperforms the latter, which has linear computational complexity in the number of users. The latter encoder in turn has been shown to outperform state-of-the-art.

\section{REFERENCES}

[1] D. Tse and P. Viswanath, Fundamentals of Wireless Communication. Cambridge, U.K.: Cambridge Univ. Press, 2005. 
[2] H. Weingarten, Y. Steinberg, and S. Shamai, "The capacity region of the Gaussian multiple-input multiple-output broadcast channel," IEEE Trans. Inform. Theory, vol. 52, no. 9, pp. 3936 -3964, Sep. 2006.

[3] C. Tian, S. Diggavi, and S. Shamai, "The achievable distortion region of bivariate Gaussian source on Gaussian broadcast channel," in Proc. IEEE Int. Symp. Inform. Theory, Jun. 2010, pp. $146-150$.

[4] A. Lapidoth and S. Tinguely, "Sending a bivariate Gaussian over a Gaussian MAC," IEEE Trans. Inform. Theory, vol. 56, no. 6, pp. $2714-2752$, Jun. 2010.

[5] D. Gündüz, E. Erkip, A. Goldsmith, and H. Poor, "Source and channel coding for correlated sources over multiuser channels," IEEE Trans. Inform. Theory, vol. 55, no. 9, pp. 3927 -3944, Sep. 2009.

[6] C. T. K. Ng, D. Gündüz, A. J. Goldsmith, and E. Erkip, "Minimum expected distortion in Gaussian layered broadcast coding with successive refinement," in Proc. IEEE Int. Symp. Inform. Theory, Jun. 2007, pp. 2226 -2230.

[7] D. Gündüz and E. Erkip, “Joint source-channel codes for MIMO block-fading channels," IEEE Trans. Inform. Theory, vol. 54, no. 1, pp. $116-134$, Jan. 2008.

[8] J. Kron, D. Persson, M. Skoglund, and E. Larsson, "Closed-form sum-MSE minimization for the two-user Gaussian MIMO broadcast channel," IEEE Commun. Letters, vol. 15, no. 9, pp. 950 -952, Sep. 2011.

[9] S. Yao and M. Skoglund, "Analog network coding mappings in Gaussian multiple-access relay channels," IEEE Trans. Commun., vol. 58, no. 7, pp. 1973 -1983, Jul. 2010.

[10] N. Farvardin, "A study of vector quantization for noisy channels," IEEE Trans. Inform. Theory, vol. 36, no. 4, pp. 799-809, Jul. 1990.

[11] Y. Linde, A. Buzo, and R. Gray, "An algorithm for vector quantizer design," IEEE Trans. Commun., vol. 28, no. 1, pp. 84 - 95, Jan. 1980.

[12] P. Floor, T. Ramstad, and N. Wernersson, "Power constrained channel optimized vector quantizers used for bandwidth expansion,” in IEEE Int. Symp. on Wireless Communication Systems, 2007, pp. 667 -671.

[13] J. Karlsson and M. Skoglund, "Optimized low-delay source-channel-relay mappings," IEEE Trans. Commun., vol. 58, no. 5, pp. 1397-1404, May 2010.

[14] D. Persson and T. Eriksson, "Power series quantization for noisy channels," IEEE Trans. Commun., vol. 58, no. 5, pp. 1405 -1414, May 2010.

[15] —- "On multiple description coding of sources with memory," vol. 58, no. 8, pp. 2242 -2251, Aug. 2010.

[16] B. Hochwald, C. Peel, and A. Swindlehurst, "A vector-perturbation technique for near-capacity multiantenna multiuser communication-part II: perturbation,” IEEE Trans. Commun., vol. 53, no. 3, pp. 537 - 544, Mar. 2005.

[17] C. Windpassinger, R. Fischer, T. Vencel, and J. Huber, "Precoding in multiantenna and multiuser communications," IEEE Trans. Wireless Commun., vol. 3, no. 4, pp. 1305 - 1316, Jul. 2004.

[18] T. Berger, Rate-Distortion Theory. Englewood Cliffs, NJ: Prentice-Hall, 1971.

[19] T. Cover and J. Thomas, Elements of information theory. New York, NY: John Wiley \& Sons, 1991.

[20] S. Vishwanath, N. Jindal, and A. Goldsmith, "Duality, achievable rates, and sum-rate capacity of Gaussian MIMO broadcast channels," IEEE Trans. Inform. Theory, vol. 49, no. 10, pp. 2658 - 2668, Oct. 2003.

[21] W. Yu, "Sum-capacity computation for the Gaussian vector broadcast channel via dual decomposition," IEEE Trans. Inform. Theory, vol. 52, no. 2, pp. 754 -759, Feb. 2006.

[22] C. Peel, B. Hochwald, and A. Swindlehurst, "A vector-perturbation technique for near-capacity multiantenna multiuser communication-part I: channel inversion and regularization,” IEEE Trans. Commun., vol. 53, no. 1, pp. 195 - 202 , Jan. 2005. 
[23] Code repository, Division of Communication Systems, Linköping University. [Online]. Available: http://www.commsys. isy.liu.se/en/publications 